\title{
Establishment of a Novel Bladder Cancer Xenograft Model in Humanized Immunodeficient Mice
}

\author{
Zhen Gong ${ }^{\mathrm{a}}$ Hanzi Xu ${ }^{\mathrm{b}}$ Yiping Su${ }^{\mathrm{a}}$ Wangfei $\mathrm{Wu}^{\mathrm{c}}$ Lin Hao ${ }^{\mathrm{d}}$ Conghui Han ${ }^{\mathrm{d}}$ \\ aDepartment of Gynecology and Obstetrics, Nanjing Maternity and Child Health Care Hospital Affiliated \\ to Nanjing Medical University, Nanjing, ${ }^{b}$ Department of Radiation Oncology, Jiangsu Cancer Hospital \\ Affiliated to Nanjing Medical University, Nanjing, 'Department of Pathology, Nanjing Maternity and \\ Child Health Care Hospital Affiliated to Nanjing Medical University, Nanjing, dDepartment of Urology, \\ the Affiliated School of Clinical Medicine, Xuzhou Medical College, Xuzhou, P.R. China
}

\section{Key Words}

Severe combined immunodeficient mice • Bladder cancer • Animal model • Immunotherapy

\begin{abstract}
Background/Aims: The aim of this study was to develop a novel model by transplanting human bladder cancer xenografts into humanized immunodeficient mice (SCID). Methods: The animals first underwent sublethal irradiation and then were subjected to simultaneous transplantation of human lymphocytes $\left(5 \times 10^{7}\right.$ cells/mouse i.p.) and human bladder cancer cells $\left(3 \times 10^{6}\right.$ cells/mouse s.c.). Results: The xenografts developed in all 12 mice that had received bladder cancer BIU-87 cells, and the tumor specimens were evaluated histologically. All 6 model mice expressed human CD3 mRNA and/or protein in the peripheral blood, spleens and xenografts. The mean proportion of human $\mathrm{CD}^{+}$cells was $19 \%$ with a level of human IgG $532.4 \mu \mathrm{g} / \mathrm{ml}$ in the peripheral blood at Week 6 after transplant inoculation. The re-constructed human immune system in these mice was confirmed to be functional by individual in vitro testing of their proliferative, secretory and cytotoxic responses. Conclusion: The successful engraftment of the human bladder cancer xenografts and the establishment of the human immune system in our in vivo model described here may provide a useful tool for the development of novel therapeutic strategies targeting at bladder cancer.
\end{abstract}

Copyright $\odot 2015$ S. Karger AG, Basel

\section{Introduction}

Novel experimental approaches to bladder cancer have been identified, which may potentially improve the outcome of affected patients; however, investigations in humans are limited due to technical and ethical considerations [1]. Therefore, alternative animal models are desirable for bladder cancer research [2]. Currently, there are four major murine bladder 
tumor models available, each having its own advantages and disadvantages: (i) the chemically induced model [3]; (ii) the syngeneic model [4]; (iii) the transgenic model [5]; and (iv) the xenograft model. The first two animal models need prolonged incubation periods and have variable reproducibility or unsatisfactory tumor take rates. The transgenic models, however, not only influence background genetic expression, but also decrease cellular heterogeneity, which in turn can affect tumor progression and metastasis [2].

As interactions between the immune system and malignant cells play an important role in tumorigenesis and cancer progression, deficiency of the immune system in the detection and rejection of transplanted tumor cells may lead to cancer development [6]. Over the years, multiple attempts have been made to activate immune responses in cancer patients to improve the therapeutic outcomes because these responses provide anti-tumor effects [7] However, one of the important disadvantages in commonly-used xenograft models (i.e., nude mouse model) is the absence of a complete immune system so that these animals lack crucial components including functional $\mathrm{T}$ and $\mathrm{B}$ lymphocytes in the immune deficient hosts. So far, no ideal animal models for bladder cancer research have been identified.

Compared to a nude mouse model, which only hosts B lymphocytes, the severe combined immunodeficient (SCID) mouse model lacks mature T and B lymphocytes. This specific property has allowed animal model research to move forward [8]. After the first "humanized" model was developed [9] by transplanting human lymphocytes into SCID mice, a number of similar models have been studied [10-13]. Recently, it has been reported that xenografts can be transplanted simultaneously into humanized SCID mice [14-16], enriching the potency of xenograft tumor models with immune competency. We therefore hypothesized that the transplantation of human bladder cancer xenografts into humanized SCID mice may diminish the limitations associated with previous xenograft models and provide a better model with a functional re-constructed human immune system for bladder cancer research.

\section{Materials and Methods}

\section{Cell line and culture conditions}

The human bladder cancer cell line BIU-87 was obtained from the cell bank of the Committee on Type Culture Collection of the Chinese Academy of Sciences. Cells were cultured in RPMI 1640 medium (Gibco, Carlsbad, CA, USA) supplemented with $10 \%$ fetal bovine serum (FBS; Gibco). To ensure that the tumor cells were comparable, only passages $2-4$ were used for the xenotransplantation.

\section{Animals}

C.B-17 scid/scid mice were obtained from Vital River Laboratories in Beijing, China and maintained under specific pathogen-free conditions in the animal facility of the Medical School of Southeast University in Nanjing, China. The principles of laboratory animal care and regulations for animal studies were strictly followed. Only non-leaky mice (i.e., less than $1 \mu \mathrm{g} / \mathrm{ml}$ of total murine serum immunoglobulin), aged 4-6 weeks, were used in the experiments.

\section{Transplantation}

A total of 24 animals were irradiated prior to peripheral blood lymphocyte (PBL) engraftment. A dose of 3.5 Gy radiation to each mouse was administered in the Department of Radiation Oncology, Jiangsu Cancer Hospital in Nanjing, China. These pretreated mice were then randomly distributed into 4 groups ( $\mathrm{n}=6$ mice/ group): (1) Humanized control animals were treated as described previously [17]. Briefly, PBLs from healthy donors were isolated by density gradient centrifugation using lymphocyte separation medium (Axis-Shield PoC AS, Oslo, Norway), and then they were intraperitoneally injected $\left(5 \times 10^{7}\right.$ cells $/$ mouse $)$ into SCID mice under sterile conditions. (2) Xenograft control animals were developed as described previously [18]. Briefly BIU-87 cells were trypsinized and resuspended in RPMI-1640 medium. Approximately $3 \times 10^{6}$ cells were subcutaneously transplanted into the hind legs of the animals. (3) Model animals were then bearing PBL engraftment (i.p.) and subsequent BIU-87 cell xenotransplantation (s.c.). (4) Blank control animals were then injected with an equivalent amount of PBS (i.p. and s.c.). 


\section{Cellular Physiology Cell Physiol Biochem 2015;37:1355-1368 \begin{tabular}{l|l} 
and Biochemistry & $\begin{array}{l}\text { DOI: 10.1159/000430401 } \\
\text { Published online: October 22, } 2015\end{array}$ \\
\hline
\end{tabular} \\ Gong et al.: Humanized Mice Model for Bladder Cancer}

\section{Observation of biological features}

The animals were continuously observed and clinically examined until the $8^{\text {th }}$ week before sacrifice, and the diagnostic criteria for graft-versus-host disease (GVHD) were based on the existing literature [19].

\section{BIU-87 xenograft determination}

The xenograft growth was monitored weekly and quantified by ultrasound imaging (The MicroMaxx®) ultrasound system, SonoSite, USA) [20]. The longest dimension (D) and the perpendicular width (d) were determined, and the tumor volume $(\mathrm{v})$ was estimated by the formula: $\mathrm{D} \times \mathrm{d}^{2} / 2$. The mice were sacrificed at the end of the $8^{\text {th }}$ week, and the tumors were excised for macroscopic examination, a real-time PCR and immunohistochemical (IHC) study.

\section{Assessment of human PBL engraftment}

Determination of human immunoglobulin levels in mice. Serum samples from individual mice were screened for the presence of human immunoglobulins (HuIg) using a commercial ELISA kit (MultiSciences Biotech, Hangzhou, China), and the whole process was performed according to the manufacturer's instructions.

Assessment of T lymphocyte invasion in mice. Real-time PCR: After the mice were sacrificed, total RNA was isolated from RBC-depleted peripheral blood, the spleen and xenograft tissues by employing the Invitrogen RNA extraction kit. The RNA was quantified subsequently with spectrophotometry. The firststrand complementary DNA was synthesized from 500 ng of total RNA, and quantitative PCR measurements were performed with TBP as a passive reference using the Rotor-Gene system. The conditions for PCR were recorded as follows: $2 \mathrm{~min}$ at $50^{\circ}$ and $10 \mathrm{~min}$ at $95^{\circ}$ for one cycle followed by 10 seconds at $95^{\circ}$ and 30 seconds at $60^{\circ}$ for 50 cycles. The sequences of the endpoint primers for the human T lymphocyte CD3 marker were recorded as follows: forward: 5'-TCC TTG CTG TTG GGG TCTAC-3'; reverse: 5'-GGT CAT TGG GCA ACA GAGTC-3', and the sequences of the TBP primers were logged as follows: forward: 5'-TTC GGA GAG TTC TGG GATT-3'; reverse: 5'-GAA AAT CAG TGC CGT GGTTC-3'. Only one single product of approximate $100 \mathrm{bp}$ was detected during the melting curve analysis following qRT-PCR (Fig. 2B, 2C), indicating that the amplified product specifically corresponded to human CD3 mRNA. The REST-2009 program was used to calculate the relative changes in human CD3 mRNA compared with the housekeeping gene (TBP) [21].

Immunohistochemical staining: The remaining tissues of the spleen and xenograft tissues were fixed in $4 \%$ formalin and paraffin embedded after proper processing. Sections were cut, and the tissues were deparaffinized with xylol and ethanol. Antigen retrieval was performed by using heat application in citrate buffer. The slices were blocked with $1 \%$ FCS and incubated overnight at $4^{\circ} \mathrm{C}$ with MAbs against human CD3. On the following day, antibody binding was revealed using the ABC Kit, and the color further developed with a DAB kit (KeyGen Biotech, Nanjing, China). The slides were counterstained with hematoxylin and eosin (H\&E), and photographs were taken under standard light microscopy.

Flow cytometry: Blood was drawn weekly for the first 6 weeks after engraftment. Single cell suspensions were prepared and incubated with hypotonic saline prior to flow cytometry. After being labeled with an antibody specific for human cell surface antigen CD3 at $4^{\circ}$ for $20 \mathrm{~min}$, the cells were analyzed by using flow cytometry equipped with Cell Queste software. An irrelevant isotype-matched antibody was used as a negative control in each labeling experiment. Expression was quantified by means of comparison with control staining of matched samples.

Functional analysis of human PBL engraftment

CCK-8. To evaluate the proliferation profile of human lymphocytes in the model mice, the assay was performed with a cell counting kit 8 (CCK-8, Dojindo, Kumamoto, Japan). RBC-depleted PBLs isolated from the mice in model group were aliquoted briefly into 96-well plates in 10\% FBS medium supplemented with $20 \mu \mathrm{g} / \mathrm{ml}$ phytohemagglutinin (PHA). Medium $+20 \mu \mathrm{g} / \mathrm{ml}$ PHA and PBLs incubated without PHA were used as blank and negative control wells, respectively, whereas human PBLs from healthy donors served as a positive control group. After $72 \mathrm{~h}$ incubation, $10 \mathrm{ml}$ CCK- 8 solution was added to each well, and incubation lasted for 4 more hours. The absorbance was measured with a microplate reader at the wavelength of 450 $\mathrm{nm}$. Cell proliferation was reported as the proliferation index (PI), where PI = absorbance value in the experimental wells / absorbance value in the control wells.

ELISA. To evaluate cytokine released by human lymphocytes in the model mice, culture supernatants were collected after stimulation with PHA and centrifuged once to remove particulate material. Interleukin-2 
(IL-2) was assayed using a commercial ELISA kit (ADL, San Diego, USA).

Flow cytometry. Cytotoxicity was measured at a 10:1 effector: target cell ratio using a flow cytometric CFSE $/ 7-A A D$ cytotoxicity assay and was expressed as $\%$ specific lysis $=\left(\left[\mathrm{CFSE}^{+} 7-\mathrm{AAD}^{+}\right] / \mathrm{CFSE}^{+}\right) \times 100$. The effector PBLs were co-cultured at a density of $6 \times 10^{5}$ cells per well in six-well plates with BIU-87 cells prelabeled with $1 \mathrm{mM}$ CFSE. After $72 \mathrm{~h}$, the BIU-87 cells were harvested from cultures and washed in PBS. The cells were then resuspended in binding buffer containing $20 \mathrm{mg} / \mathrm{ml}$ 7-AAD and incubated in the dark at $4^{\circ}$ for $20 \mathrm{~min}$. Acquisition was analyzed immediately by using flow cytometry as described previously [22].

\section{Statistical analysis}

All in vitro experiments were performed in triplicate, and the experiments were repeated at least three times. Numerical data were presented as the mean $\pm S D$, and a statistical analysis was performed with Student's t test or a one-way ANOVA followed by Dunnett's multiple comparison as appropriate. A two-way ANOVA followed by Tukey's test was also used to determine the significance of differences in the growth of xenografts and human PBL engraftment in mice. Statistical comparisons were performed using R-2.14.1 for Windows (www.r-project.org). A $P$ value of less than 0.05 was considered significant.

\section{Results}

\section{Establishment of human bladder cancer xenografts}

BIU-87 is a cell line derived from human transitional cell carcinoma (TCC) initially prepared at Peking University in China [23]. It has been widely used in studies both in vitro and in vivo in China (i.e. xenograft development) [24, 25]. Using techniques hitherto developed, xenografts in SCID mice were established by subcutaneous xenotransplantation with these cells.

The general aspects of the mice, i.e., health status, average weight and fur status, were observed daily, and the results did not differ among the four groups. As the humanized mice were chimeras, they were significantly more susceptible to GVHD. However, having engrafted with PBLs of less than $5 \times 10^{7} /$ mouse, we had no GVHD or unexpected deaths observed before sacrifice in all 24 mice.

The tumor volume between the xenograft control group and model group after plantation was measured by means of ultrasound. At the time of sacrifice, no xenografts developed in humanized or blank control mice. In contrast, all mice from xenograft control and model groups developed tumors (Fig. 1A, D), which were further investigated through macroscopic histology staining. Because no specific immunohistological antibodies were available for the cell line we used, here we provided only the representative histological photograph with HE staining. (Fig. 1C, F)

Approximately 6-7 days after xenotransplantation, tumors were detectable under ultrasound in xenograft control animals. The mean tumor volume of $2490.8 \pm 310.0 \mathrm{~mm}^{3}$ within 6 weeks of the initial appearance was recorded (Fig. 1A, B). Interestingly, the model animals showed much slower rates of tumor growth with significantly smaller volumes at the time of sacrifice $\left(1116.3 \pm 150.1 \mathrm{~mm}^{3}\right.$, Fig. 1D, E, $\left.p<0.05\right)$.

\section{Human PBL engraftment in model mice: Serum from the model mice contained $T$ and $B$} lymphocytes

To evaluate the possible engraftment of $T$ and $B$ lymphocytes, serum samples from individual mice were monitored for the presence of HuIg and human CD3 mRNA after sacrifice. Consistent with a previous report [14], all humanized animals produced detectable levels of HuIg. The model mice had mean serum HuIg levels in excess of $500 \mu \mathrm{g} / \mathrm{ml}$, which were significantly higher than that in the humanized control mice $(\mathrm{P}<0.005)$. Animals without PBLs, including the blank and xenograft control mice, did not produce detectable levels of HuIg (Fig. 2A).

Moreover, quantification of serum CD3 mRNA revealed positive expression in the serum of model and humanized control mice, but not in that of blank or xenograft control ones (Fig. 


\section{Cellular Physiology Cell Physiol Biochem 2015;37:1355-1368 and Biochemistry

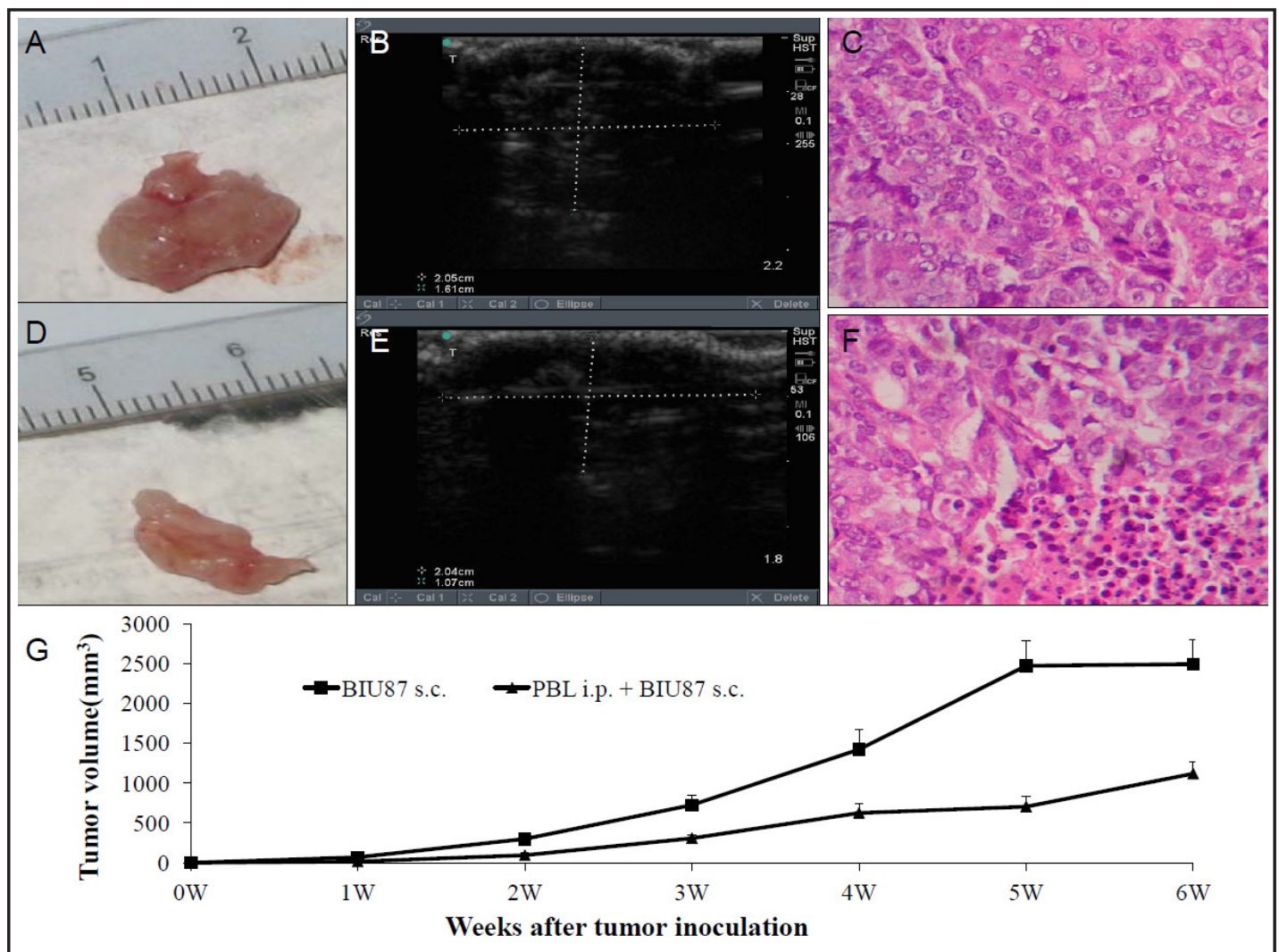

Fig. 1. Growth of human bladder cancer BIU-87 xenografts in mice. All mice were injected with BIU-87 cells s.c. and/or with PBLs i.p. The tumors were monitored by ultrasound imaging, and at sacrifice, the tumors were harvested for imaging and H.E. staining. (A, B, C) are representative images of a harvested tumor, ultrasound imaging and H.E. staining in a xenograft control mouse, respectively; (D, E, F) represent the corresponding results in model mice. $(G)$ indicates the growth curve after quantification. Statistical analysis was performed using two-way ANOVA followed by Tukey's test, and the difference between the xenograft control and model mice was significant $(p<0.05)$.

2D), indicating lymphocyte infiltration into the model mice and humanized control mice serum.

Human PBL engraftment in model mice: T lymphocytes infiltrated into the spleens of the model mice

To characterize the T lymphocyte population in the spleens, the tissues were weighed, and real-time PCR and immunohistochemical staining were performed to analyze CD3 mRNA and protein in all individuals. Consequently, the weight of spleen in the xenograft control mice was approximately $50 \mathrm{mg}$, which was slightly higher than that in the blank control mice but still significantly lower than those in the humanized control and model mice (Fig. 3AE). Similarly, human CD3 mRNA and $\mathrm{CD}^{+}$cells were detectable by using qRT-PCR and IHC staining in the humanized control and model mice, but not in the blank and xenograft control mice (Fig. 3F-J), suggesting the presence of T lymphocyte infiltration into the spleen of the model mice. Interestingly, no significant correlation between the spleen weight and the level of the CD3 mRNA was identified between the humanized control mice and the model ones (Fig. 3E-F).

Human PBL engraftment in model mice: T lymphocytes penetrated into the model mice xenografts

Because the above data suggested the infiltration of $\mathrm{T}$ lymphocytes into serum and spleens of the model mice, the potency of penetration into the xenograft was further KARGER 


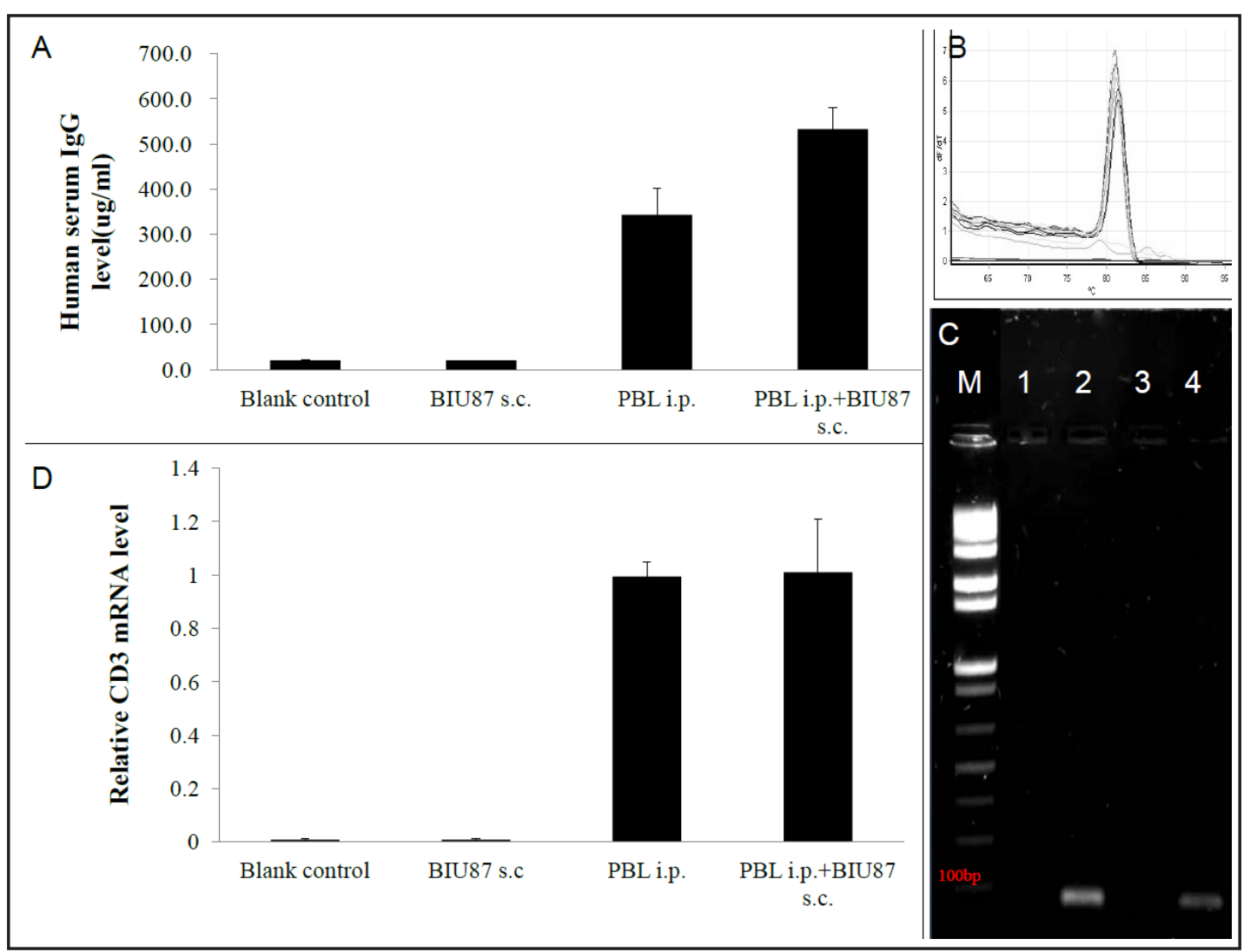

Fig. 2. Serum from the model mice contained T and B Lymphocytes. Six weeks after transplantation, the mice were sacrificed and the serum was harvested for ELISA assay and qRT-PCR examination. (A) shows the HuIg concentrations measured in the serum after sacrifice at week 6. (B and C) are the representative images of the melting curve and SDS-PAGE of the CD3 mRNA qRT-PCR products. (D) shows the relative expression of serum CD3 mRNA in mice. Statistical analysis was performed by one-way ANOVA followed by Dunnett's multiple comparison. The model mice produced significantly higher levels of serum $\operatorname{HuIg}(\mathrm{P}<0.05)$, but they did not show higher CD3 mRNA expression $(P \geq 0.05)$ than the humanized control mice. Meanwhile, the blank and xenograft control mice did not show any HuIg and CD3 mRNA after sacrifice.

examined after sacrifice. In contrast to the control xenograft mice, despite the presence of human CD3 mRNA in the model mice, IHC staining for CD3 was also positive in these xenograft tissues at a later point of time (Fig. 4A-E). This provided evidence that a number of human T lymphocytes had penetrated into the xenografts in the model mice.

\section{Detailed development of human PBL engraftment in the model mice}

Although these results primarily demonstrated the successful establishment of a BIU87 xenograft in humanized SCID mice, the detailed development of human PBLs in mice was unknown during the 6-week engraftment. Therefore, we quantified the HuIg and CD3 ${ }^{+}$ cell contents in peripheral blood taken in advance weekly from the mice during the 6-week engraftment by means of ELISA assays and flow cytometry. The model mice had substantial levels $(\geq 100 \mu \mathrm{g} / \mathrm{ml})$ of HuIg in their sera one week after PBL inoculation, and the changes were expressed as a gradually increasing trend corresponding to the inoculation time. The humanized control mice, on the other hand, did not start to have the same amount of HuIg until much later, i.e., 3-4 weeks after PBL inoculation. Among blank and xenograft control mice, however, no HuIg was detected at any of the five time intervals (Fig. 5A).

The engraftment of $\mathrm{CD}^{+}$cells was relatively poor during the initial 3 weeks after transplantation (Fig. 5B). Unexpectedly, as the inoculation continued, the content of $\mathrm{T}$ 


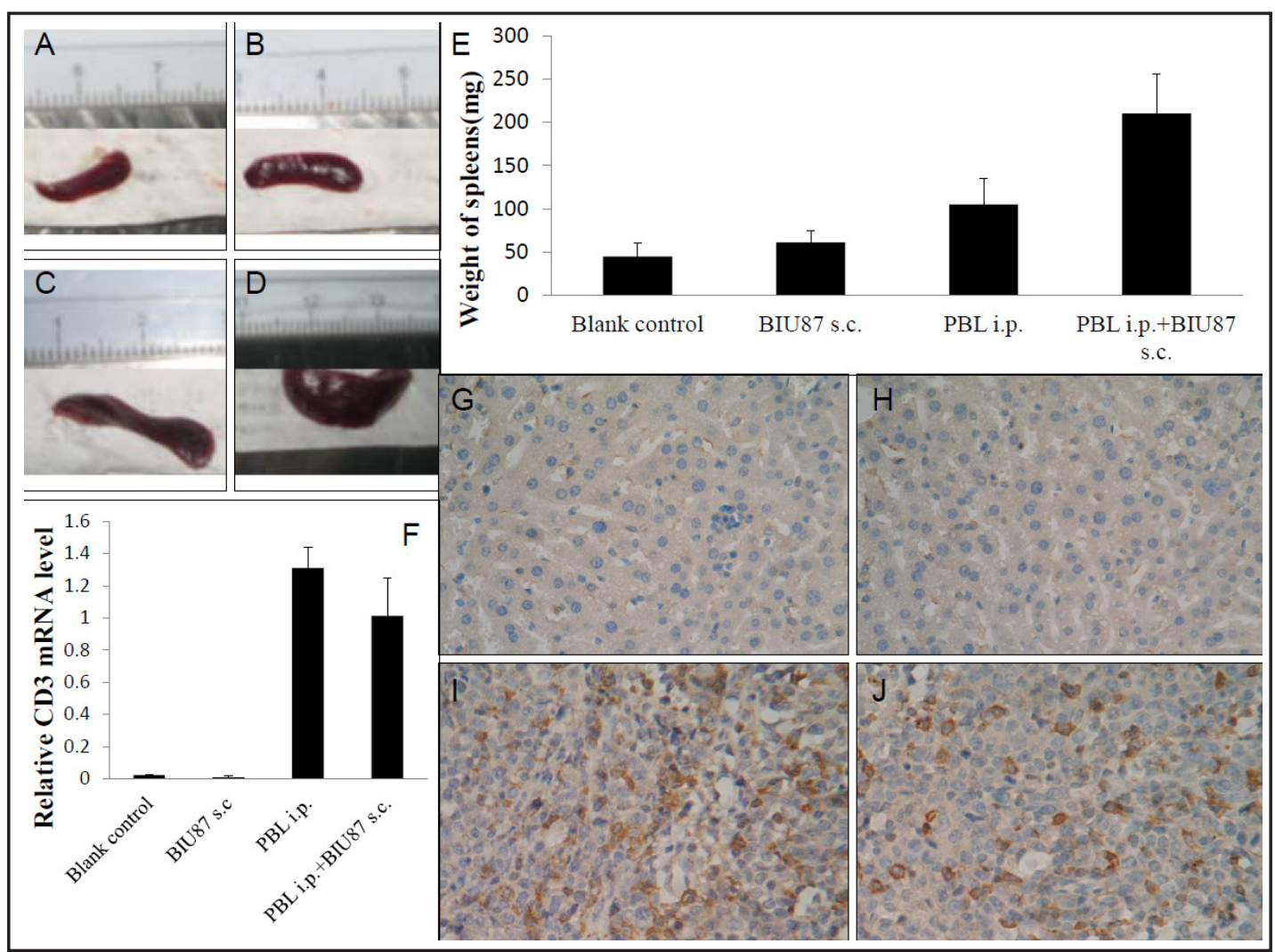

Fig. 3. T lymphocytes infiltrated the spleens of the model mice. After sacrifice, spleen tissues were harvested and submitted to qRT-PCR and immunohistochemical staining. (A-D) are the representative images of the excised spleens in blank control (A), xenograft control (B), humanized control (C) and model (D) mice. Figs. E and F show the weights of mice and the human CD3 mRNA levels in the mice normalized by the model mice. Statistical analysis was performed by one-way ANOVA followed by Dunnett multiple comparison, and the differences between controls and the model were significant. (G-H) indicate the representative expression of $\mathrm{CD}^{+}$(brown color) cells in the blank control $(\mathrm{G})$, xenograft control $(\mathrm{H})$, humanized control (I) and model (J) mice.

lymphocytes in the serum from the model mice increased to nearly $20 \%$ at 6 weeks, which is slightly higher than that in the humanized control mice $(P \geq 0.05)$. In the blank and xenograft control mice, again, there were no $\mathrm{CD}^{+}$cells detected at any time before sacrifice (Fig. 5B).

Overall, it can be concluded that both $\mathrm{T}$ and B lymphocytes developed differently after PBL engraftment in model mice.

Function of human lymphocytes in model mice

As reported in the existing literature, a variety of assays have been employed to assess lymphocyte functions. It was of great interest to us to be able to determine the extent of lymphocyte proliferation in the current study. Therefore, the complementary approach of CCK8 assay was carried out. As shown in Fig. 6A, the proliferation profile of human lymphocytes in the serum of the model mice was comparable to that in healthy donors after PHA boosting $(P \geq 0.05)$, indicating that these human lymphocytes can be stimulated to proliferate in response to PHA.

Considering that there has been no evidence showing that BIU-87 secretes any human IL-2, an IL-2 release experiment was performed using culture supernatants from the above experiments instead of using the model mice serum. Unexpectedly, the lymphocyte's ability to release cytokines was impaired (as shown in Fig. 6B), as the level of human IL2 in the model mice was significantly lower than that in healthy donors. 


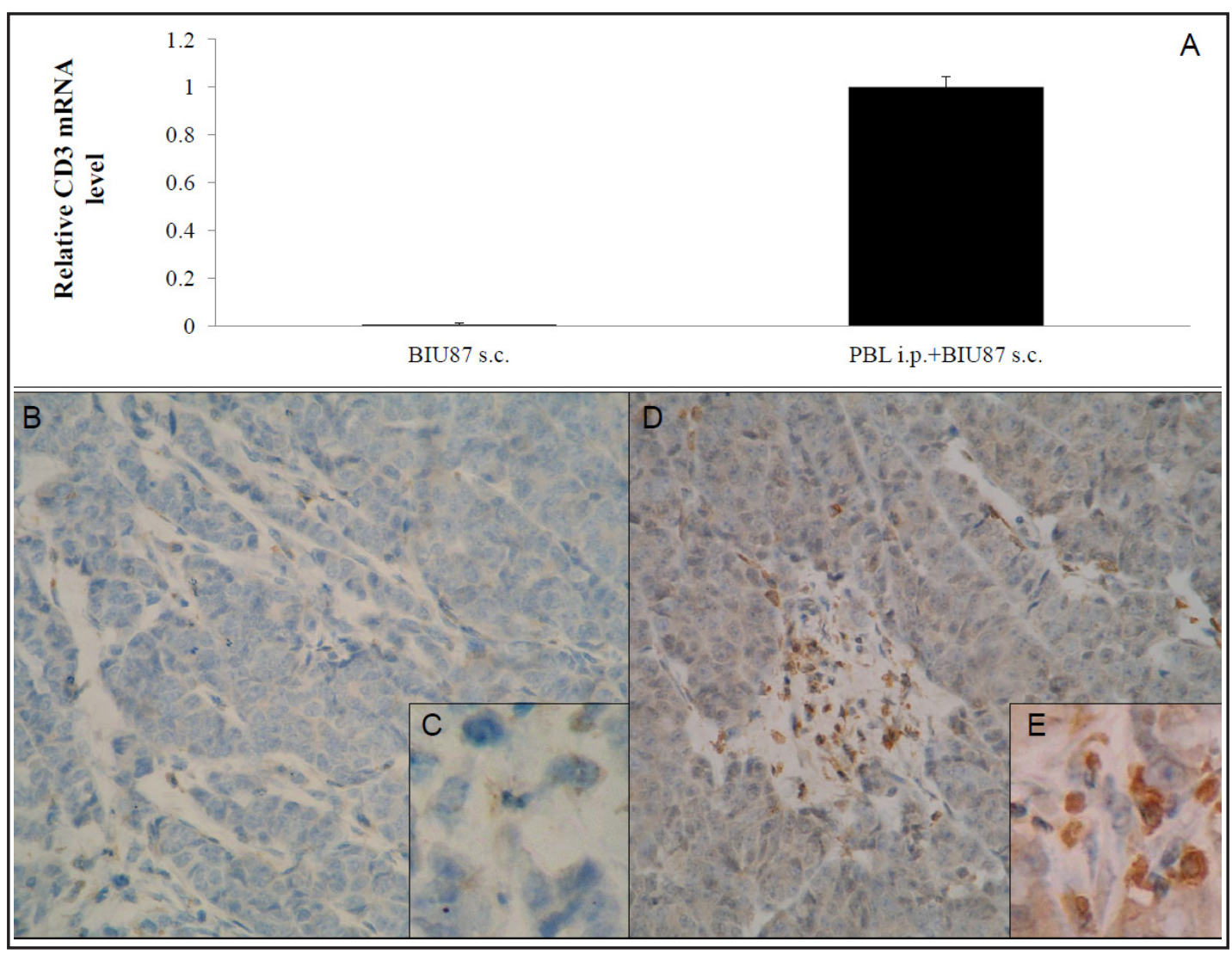

Fig. 4. T lymphocytes penetrated into model mice xenografts. After sacrifice, qRT-PCR and immunohistochemical staining were performed to analyze the differences in the levels of human CD3 mRNA, and the results were normalized by the model mice (A) to compare the representative $\mathrm{CD}^{+}$(brown color) cells (BE) in the xenograft between the xenograft control mice $(B=\times 100, C=\times 400)$ and the model mice $(D=\times 100$, $\mathrm{E}=\times 400$ ). Statistical analysis was performed with Student's $t$ test, and the differences between the xenograft control and model were significant.

It was also of interest to examine the function of these cells by measuring in vitro lysis of sensitive targets. Since BIU-87 cells are sensitive to human lymphocytes [22], these cells were used in the present experiments. Consequently, human lymphocytes from the model mice displayed a lysis response of BIU-87 at levels similar to that of normal human PBLs (mean rate of lysis with $4.8 \%$ vs. $6.1 \%$ (as shown in Fig. 6C-E).

\section{Discussion}

Although bladder cancer greatly affects patients' well-being [26], its intricate biology is poorly understood. New therapeutics targeting at bladder cancer are emerging [27]; however, their progress is hindered by limitations in the scope of in vitro experimentation. Therefore, there has been a growing demand for animal models that are able to sustain studies of bladder cancer in vivo [2].

In theory, an ideal tumor model should allow tumors to grow in locus, but in practice, orthotopic models for bladder cancer are severely restricted by technical difficulties. The procedures to produce such models are time-consuming and tumor uptake is often poor [28]. Among the existing orthotopic models, pretreatment with poly-L-lysine and trypsinoptimized orthotopic bladder tumor implantation is required to improve bladder tumor uptake [29]. Unexpectedly, the tumor size is still extremely limited due to the small murine bladder capacity [29, 30].

\section{KARGER}




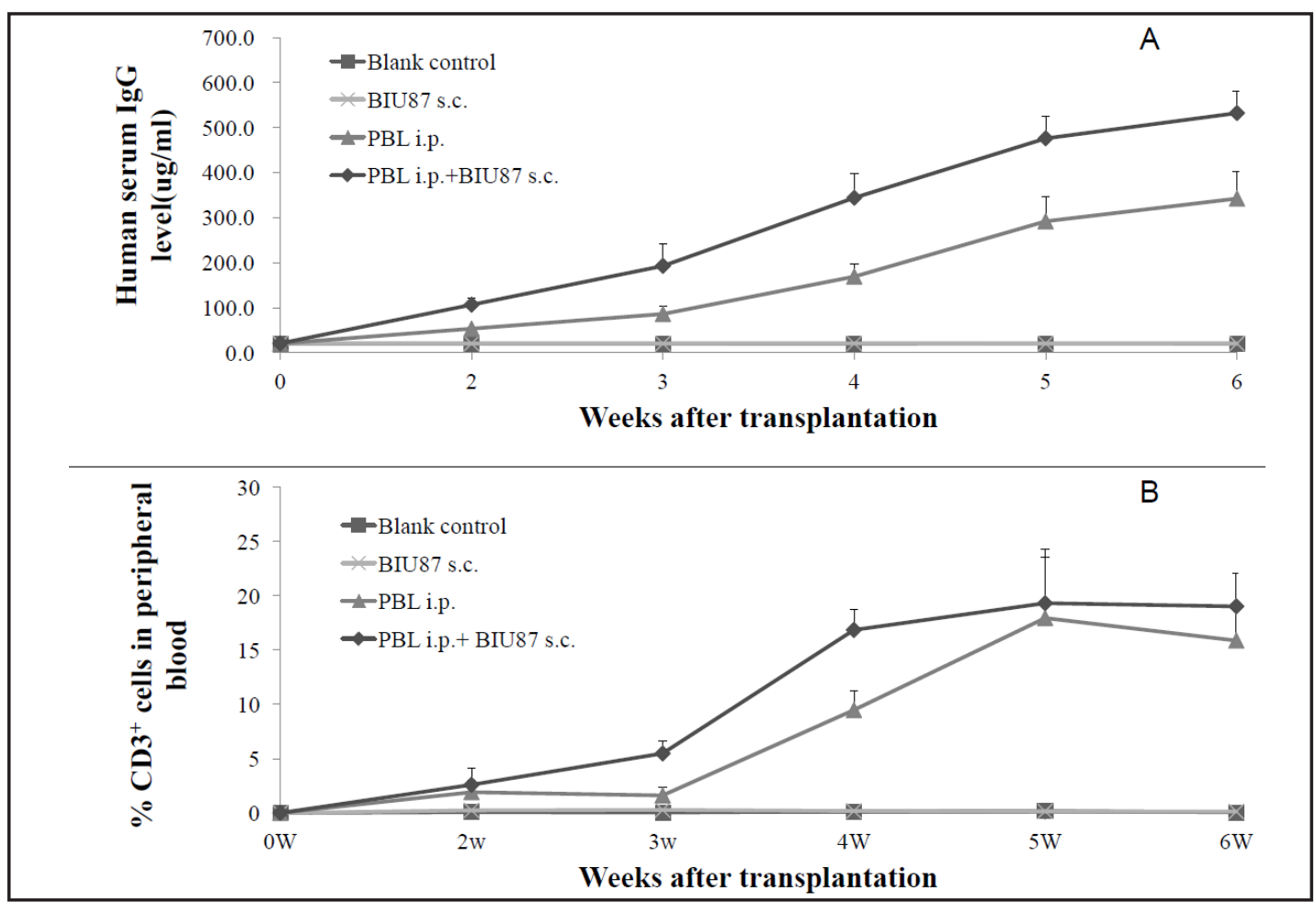

Fig. 5. Detailed development of human PBL engraftment in the model mice. Mice peripheral blood was harvested weekly during the 6-week engraftment, and the HuIg levels (A) and CD3 ${ }^{+}$cell contents (B) were quantified by ELISA assay and flow cytometry, respectively. Statistical analysis was performed by two-way ANOVA followed by Tukey's test, and the difference of HuIg levels between controls and model was significant (No difference in the CD3+ cell content between the humanized control and model was found.

Though failing to provide an organ-specific tumor microenvironment, subcutaneous models have been widely used, because they facilitate tumor treatments, monitoring and generate good tumor size [30]. In this study, we chose to use the subcutaneous xenograft to investigate the bladder cancer model we established.

To develop a subcutaneous xenograft, immunodeficient nude mice are commonly used, thereby leading to a major disadvantage of a deficient immune response in these models. Compared to nude mice, SCID mice are profoundly lacking in functional, mature $\mathrm{T}$ and $\mathrm{B}$ lymphocytes, and are more suitable for xenotransplantation [31]. Moreover, they are suitable hosts for the engraftment of human immunocompetent cells (humanized SCID mouse) [9-13], resulting in a somewhat more relevant microenvironment [30]. These animals not only are ready to accept grafts of human immunocompetent cells to develop humanized SCID mice, but they are also able to host malignant human tumors to develop subcutaneous xenografts. As such, if the engraftment of these two functionally varied cell types to the same animal is successful, it would be possible for us to develop an in vivo chimera model. In particular, this model is able to overcome the immunodeficiency issues of the other models using nude mice xenograft [14-16].

In fact, the success of engrafting human PBLs into SCID mice has been varied in different laboratory reports [32], which may be attributed to a range of factors, such as graft issues and host situations. In this context, a series of potential factors were considered in our study.

For example, C.B.17 SCID mice contain innate immune cells, i.e., undeleted natural killer (NK) cells [8,33], and these may present a problematic barrier to immunocompetent grafts. To overcome this barrier, the reduction of interference by the NK cells is extremely desirable. To date, there have been multiple published data demonstrating that the pretreatment similar to what we used could achieve significant efficiency of engraftment. For example, 


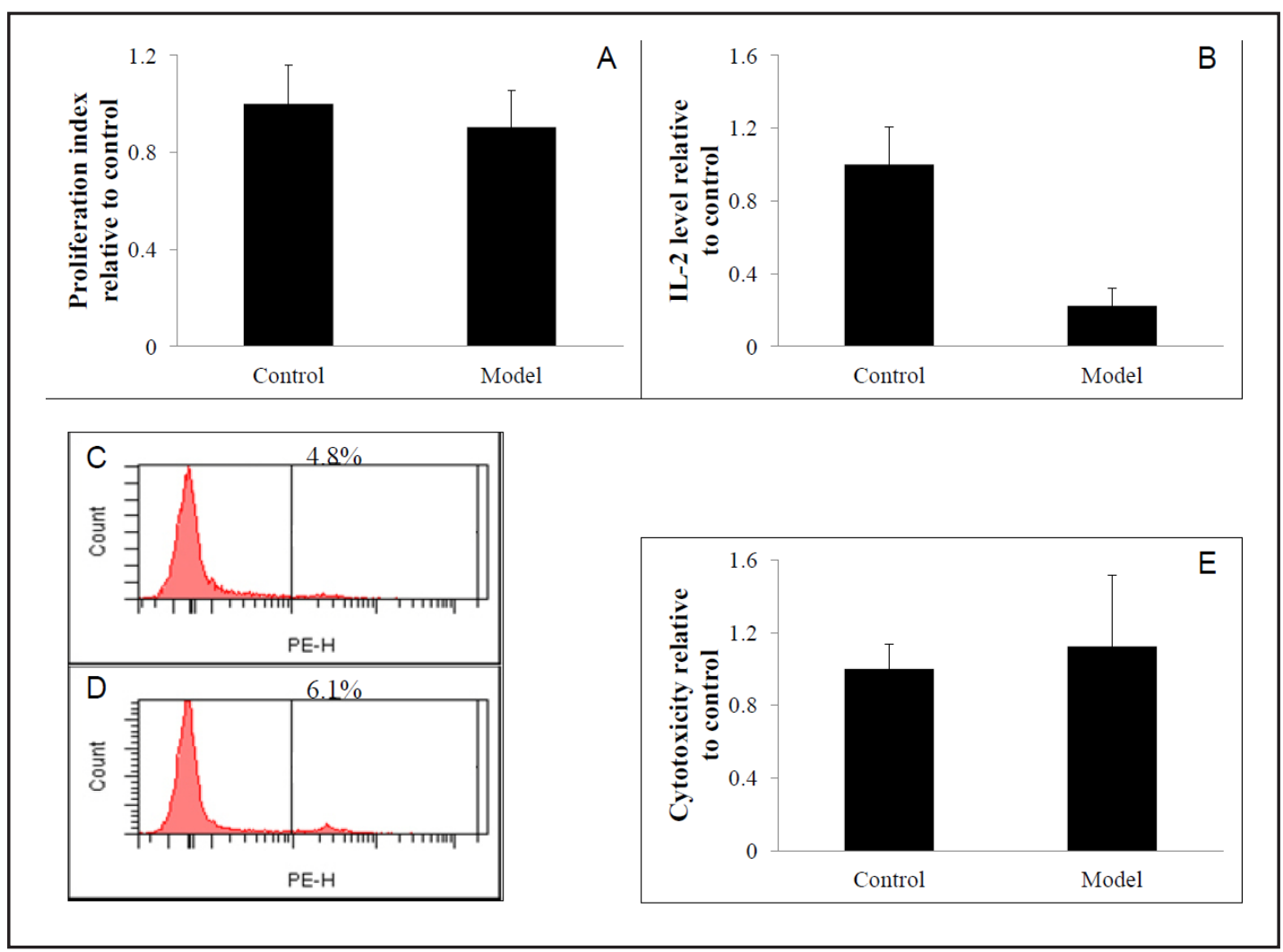

Fig. 6. Function of human lymphocytes in model mice. RBC-depleted PBLs isolated from the model mice (Model) or healthy donors (Control) were used in the CCK8 assay for the analysis of PBL proliferation. The proliferation index was normalized by the control, and the result is shown in (A). The culture supernatants were harvested and used for the IL-2 release ELISA assay. The corresponding IL-2 levels were normalized by the Controls and are shown in (B). The lysis observed by FCM for the Control (C) and Model (D) mice are quantified in (E). Statistical analysis was all performed using the Student's t test, and the differences between the xenograft controls and models were significant for IL-2 level $(B, P<0.05)$, but not for Proliferation or Cytotoxicity (A and B, P $\geq 0.05$ ).

Hochman et al. have shown that sub lethal irradiation markedly reduced murine NK activity [34]. Barry et al. have indicated that such a pretreatment also improved human engraftment from $37 \%$ to $83 \%$ [35]. Shpitz et al. have shown that their reconstituted mice did not attract GVHD after radiation pretreatment, which led to better survival of the mice [33]. Seitz et al. recently developed a humanized mouse model of childhood rhabdomyosarcoma within the SCID mice only underwent sublethal irradiation [15]. Thus, in our study, a dose of 3.5 Gy radiation was administered prior to PBL transplantation. As we were mainly focused on increasing the engraftment of PBLs in the current study, experiments to compare the tumorigenesis with or without irradiation pretreatment were not conducted.

Moreover, the tissue origin of the reconstituted human immune system and the construction approach were also taken into account. So far, there are a variety of tissues being used in mice humanization, including fetal thymus, umbilical cord blood cells, hematopoietic stem cells, PBLs and spleen cells [9-13]. Because PBLs are easy to isolate, they are more commonly used for being economically appropriate. We also used these cells in the establishment of our model. Among the various transplantation methods, intraperitoneal injection or tail vein injection has been typically applied with intraperitoneal injection (i.p.), which was more readily manipulated. Taking all this into consideration, we therefore made efforts to humanize SCID mice with the engraftment of PBL cell via i.p. route.

Based on previous studies [36] and our initial trials, $5 \times 10^{7} \mathrm{PBL}$ cells per mouse were transplanted into animals for the purpose of increasing the success rate of "humanization" 


\section{Cellular Physiology Cell Physiol Biochem 2015;37:1355-1368 \begin{tabular}{l|l} 
and Biochemistry & $\begin{array}{l}\text { DOI: 10.1159/000430401 } \\
\text { Published online: October 22, } 2015\end{array}$ \\
\hline
\end{tabular}

and minimizing the possibility of GVHD. Consequently, no GVHD was observed in any of the animals in our study.

The cluster of differentiation (CD) is commonly used as cell markers in immunophenotyping. Although CD4 and CD8 are commonly used as markers for assessing helper and cytotoxic $\mathrm{T}$ cells respectively, these molecules are defined in combination with CD3+ (i.e. $\mathrm{T}$ helper cell markers are CD45+, CD3+, CD4+; Cytotoxic $\mathrm{T}$ cell markers are $\mathrm{CD} 45+, \mathrm{CD} 3+, \mathrm{CD} 8+$ ] [37]. Many researchers have chosen CD3 as the cell marker for the $\mathrm{T}$ lymphocyte detection [38]. For example, anti-CD3 monoclonal primary antibody was used for T-lymphocyte detection in the recent two studies with good sensitivity in measurement $[39,40]$. Hence, we decided to choose CD3 as a marker for the T lymphocytes infiltration.

Consequently, HuIg and human CD3 mRNA, protein were only detected in the humanized mice (including the model mice and the humanized control mice, as shown in Fig. 2A-D). We observed that the HuIg concentrations were a bit lower in humanized control mice than that in model mice $(p<0.05)$. Meanwhile, CD3 mRNA expression of humanized control mice was comparable with that of the model mice. We believed that the serum infiltration of human $\mathrm{T}$ and B cells was possibly different between the groups (humanized control vs model).

In general, a large number of different types of functional immune cells are located in the spleen. Therefore, we examined the existence of human PBLs in the spleen to further evaluate our establishment of the engraftment. We again found the enlargement of the spleen, and the human CD3 mRNA and molecular markers were detected in the humanized mice, especially in the model mice (Fig. 3A-J). These experiments clearly demonstrated that the spleens of the model mice were successfully engrafted with human PBLs.

These results represent our successful establishment of a novel xenograft model of human bladder cancer with BIU-87 cells in the humanized mice. Their tumor growth exhibited a characteristic pattern. However, the penetration of the engrafted PBLs into the subcutaneous xenograft has not been explored in the previous studies of the humanized SCID mouse models bearing subcutaneous tumors. We then investigated the possibility of eliciting a specific immune response to growing tumors within these animals. For the first time, we discovered that transplanted PBLs penetrated into the subcutaneous xenografts, as human CD3 mRNA and CD3-positive cells were both detected in these xenografts (Fig. 4A-E).

We hope that not only the human lymphocytes were engrafted into the tumor xenograft of model mice, but their functions were also fully preserved, if this model is to be used in studies of cancer immunotherapy. Because it has been shown that several cytokines, such as IL-4, IL-5, IL-2, IFN- $\gamma$, were detectable in SCID mice transplanted with human PBLs [41], we only included IL-2 as the representative cytokine in the current study. We found that the isolated PBLs from the serum of model mice did produce certain cytokines, i.e., IL-2 (Fig. 6B) and they also maintained the proliferating profile (Fig. 6A), representing the underlying immune responses were retained.

Because these cells, together with the cytokines produced, may be responsible for the inhibition of xenograft growth, we tested their cytotoxicity against TCC BIU-87 cells. The results showed that similar to PBLs from healthy donors, these PBLs also reacted to the xenograft BIU-87 cells and contributed to their lysis (Fig. 6C-E). Therefore, it is more likely that the functions of PBLs transplanted into model mice were preserved to a certain extent.

Iwanuma et al. [42] have confirmed that by s.c. coengraftment of PBLs and tumor into SCID mice, PBLs suppress the growth of tumor xenograft; and the tumor suppression was dependent upon T lymphocytes. Sabzevari H et al. [43] demonstrated that when engrafted with T lymphocytes, SCID mice bearing tumors showed a significant reduction of metastasis in the number of foci and in the lymph nodes. Liu et al. [6] found the xenograft growth in SCID mice was significantly inhibited by intraperitoneal injection of PBMCs. So they concluded that "PBMC transplantation inhibits lung carcinoma progression via the reconstitution of the immune system, particularly of cytotoxic T lymphocytes."

In our study, we first showed: (1)the model mice had high levels of HuIg and human PBL T cells infiltration and (2)the xenografts growth was inhibited (Fig. 1G). These results provided the initial evidence of the potential occurrence of immunity-mediated tumor suppression.

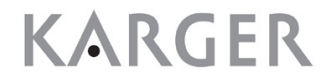


Additionally, we showed that transplanted PBLs penetrated into the xenografts of the model mice (Fig. 4A-E). These results provided a second level of evidence demonstrating the underlying interaction between the subcutaneous tumor and the reconstructed immune system in model mice. Finally, we showed that the PBLs infiltrated into the xenograft and maintained their function (Fig. 6A-E), thus offering further evidence for the possible tumor suppression of humanized immune response in model mice.

In view of the above observations, we hypothesize that reduced tumor size in model group implies that engrafted lymphocytes may either inhibit the proliferation of tumor cells or induce their apoptosis. Nonetheless, so far we have no specific evidence verifying this hypothesis. Therefore, further study needs to be done. Our major goal in this study was to successfully establish and validate this model as a useful approach in cancer immunology studies, esp. to bladder cancer. However, similar to some of the related studies [6, 14-16], this study did not include any T cell functions comparison between the humanized control group and the model group. Yet, we strongly believe that it would be of great significance to our future studies to explore the possibility of interaction between the immune system and the tumors using this model.

The role of the secretary system in lymphocytes has been investigated in details in anticancer research [44]. However, it became apparent in our study that the mean secretary viability of the transplanted lymphocytes in the model mice was significantly lower than that from healthy donors. This could have resulted from the fact that the immune cells of the mice, such as NK cells, had an inhibitory influence on the viability of the transplanted PBLs in these animals. Recently, the generation of a new immunodeficient host (NOD-scid Il2ry null mice) has achieved higher levels of PBL engraftment via usual routes, providing potential for overcoming many of the existing limitations in current humanized SCID mice [45]. Therefore, new investigations into more cases seem to be necessary to verify the secretory viability of transplanted lymphocytes within NOD-scid Il2ry null mice.

In conclusion, we have generated a novel subcutaneous xenograft model of bladder cancer in humanized C.B.17 SCID mice. This study provides a promising model for further investigations into the human immune responses and for evaluating the immunotherapeutic approaches in vivo in this type of bladder cancer.

\section{Acknowledgments}

This study was sponsored by the Graduate Student Research and Innovation Program of Jiangsu Province (CX10B086Z), the Science and Technology Development Fund of the Nanjing Medical University (2012NJMU185) and the National Natural Science Foundation of China (81202007).

\section{Disclosure Statement}

All authors have no conflicts of interest to declare.

\section{References}

1 Lopez-Beltran A, Santoni M, Massari F, Ciccarese C, Tortora G, Cheng L, Moch H, Scarpelli M, Reymundo C, Montironi R: Bladder cancer: molecular determinants of personalized therapy. Curr Drug Targets 2015;16:115-124.

2 Zhang N, Li D, Shao J, Wang X: Animal models for bladder cancer: The model establishment and evaluation. Oncol Lett 2015;9:1515-1519.

3 Hsu I, Chuang KL, Slavin S, Da J, Lim WX, Pang ST, O'Brien JH, Yeh S: Suppression of ER $\beta$ signaling via ER $\beta$ knockout or antagonist protects against bladder cancer development. Carcinogenesis 2014;35:651-661. 


\section{Cellular Physiology Cell Physiol Biochem 2015;37:1355-1368 \begin{tabular}{l|l|l}
\hline DOI: 10.1159/000430401 & (C) 2015 S. Karger AG, Basel
\end{tabular} www.karger.com/cpb

4 Chan ES, Patel AR, Larchian WA, Heston WD: In vivo targeted contrast enhanced micro-ultrasound to measure intratumor perfusion and vascular endothelial growth factor receptor 2 expression in a mouse orthotopic bladder cancer model. J Urol 2011;185:2359-2365.

5 Ayala de la Peña F, Kanasaki K, Kanasaki M, Tangirala N, Maeda G, Kalluri R: Loss of p53 and acquisition of angiogenic microRNA profile are insufficient to facilitate progression of bladder urothelial carcinoma in situ to invasive carcinoma. J Biol Chem 2011;286:20778-20787.

6 Liu X, Li H, Liu J, Guan Y, Huang L, Tang H, He J: Immune reconstitution from peripheral blood mononuclear cells inhibits lung carcinoma growth in NOD/SCID mice. Oncol Lett 2014;8:1638-1644.

7 Li W, Yang Y, Ouyang Z, Zhang Q, Wang L, Tao F, Shu Y, Gu Y, Xu Q, Sun Y: Xiao-Ai-Ping, a TCM Injection, Enhances the Antigrowth Effects of Cisplatin on Lewis Lung Cancer Cells through Promoting the Infiltration and Function of CD8(+) T Lymphocytes. Evid Based Complement Alternat Med 2013;2013:879512.

8 Nonoyama S, Ochs HD: Immune deficiency in SCID mice. Int Rev Immunol 1996;13:289-300.

9 Mosier DE, Gulizia RJ, Baird SM, Wilson DB: Transfer of a functional human immune system to mice with severe combined immunodeficiency. Nature 1988;335:256-259.

10 Kern ER, Rybak RJ, Hartline CB, Bidanset DJ: Predictive efficacy of SCID-hu mouse models for treatment of human cytomegalovirus infections. Antivir Chem Chemother 2001;12:S149-156.

11 Yim YS, Noh YH, Kim DH, Lee MW, Cheuh HW, Lee SH, Yoo KH, Jung HL, Sung KW, Choi SJ, Oh WI, Yang YS, Koo HH: Correlation between the immature characteristics of umbilical cord blood-derived mesenchymal stem cells and engraftment of hematopoietic stem cells in NOD/SCID mice. Transplant Proc 2010;42:27532758.

12 Chow L, Aslam R, Speck ER, Kim M, Cridland N, Webster ML, Chen P, Sahib K, Ni H, Lazarus AH, Garvey MB, Freedman J, Semple JW: A murine model of severe immune thrombocytopenia is induced by antibody-and CD8+ T cell-mediated responses that are differentially sensitive to therapy. Blood 2010;115:1247-1253.

13 Marcheix B, Yacoub-Youssef H, Calise D, Thiers JC, Benoist H, Blaes N, Ségui B, Thomsen M, Dambrin C: Human immune reconstitution with spleen cells in SCID/Beige mice. Transplant Proc 2005;37:2888-2889.

14 Ye F, Chen H, Liang Z, Lu W, Cheng Q, Xie X: Establishment of a cervical cancer model via inoculating SiHa Cells into humanized severe combined immunodeficient mice. Eur J Gynaecol Oncol 2006;27:566-572.

15 Seitz G, Pfeiffer M, Fuchs J, Warmann SW, Leuschner I, Vokuhl C, Lang P, Handgretinger R, ArmeanuEbinger S: Establishment of a rhabdomyosarcoma xenograft model in human-adapted mice. Oncol Rep 2010;24:1067-1072.

16 Abate-Daga D, Lagisetty KH, Tran E, Zheng Z, Gattinoni L, Yu Z, Burns WR, Miermont AM, Teper Y, Rudloff U, Restifo NP, Feldman SA, Rosenberg SA, Morgan RA: A novel chimeric antigen receptor against prostate stem cell antigen mediates tumor destruction in a humanized mouse model of pancreatic cancer. Hum Gene Ther $2014 ; 25: 1003-1012$.

17 Zhou W, Ohdan H, Tanaka Y, Hara H, Tokita D, Onoe T, Asahara T: NOD/SCID mice engrafted with human peripheral blood lymphocytes can be a model for investigating B cells responding to blood group A carbohydrate determinant. Transpl Immunol 2003;12:9-18.

18 Cheng H, Luo C, Wu X, Zhang Y, He Y, Wu Q Xia Y, Zhang J: shRNA targeting PLCE inhibits bladder cancer cell growth in vitro and in vivo. Urology 2011;78:474.e7-11.

19 Dwyre DM, Holland PV: Transfusion-associated graft-versus-host disease. Vox Sang 2008;95:85-93.

20 Patel AR, Chan ES, Hansel DE, Powell CT, Heston WD, Larchian WA. Transabdominal micro-ultrasound imaging of bladder cancer in a mouse model: a validation study. Urology 2010;75:799-804.

21 Pfaffl MW, Horgan GW, Dempfle L. Relative expression software tool (REST) for group-wise comparison and statistical analysis of relative expression results in real-time PCR. Nucleic Acids Res 2002;30:e36.

22 Han C, Gong Z, Hao L, Yang J, Hu J, Dong B, Fan T, Tang W, Teng G: Mechanism of monoclonal antibodycoupled Staphylococcus superantigen-A induced apoptosis in human bladder cancer cells. Cell Biochem Biophys 2011;61:679-684.

23 Lizhang Yu, Yali Huang, Chen Yang: Establishment of human bladder-cancer cell-line BIU-87 and its biological characteristics. [Article in Chinese] J Peking University (Health Sciences) 1989:67-68.

24 Du HF, Ou LP, Yang X, Song XD, Fan YR, Tan B, Luo CL, Wu XH: A new PKC $\alpha / \beta / T B X 3 / E-c a d h e r i n$ pathway is involved in PLCE-regulated invasion and migration in human bladder cancer cells. Cell Signal 2014;26:580593.

25 Zhen G, Cong-hui H, Lin H, Jian-jun Y, Wen-hao T, Gao-jun T: Preparation and in-vitro bioactivity of a novel superantigen conjugate targeting bladder carcinoma. J Pharm Pharmacol 2009;61:869-875. 


\section{Cellular Physiology Cell Physiol Biochem 2015;37:1355-1368 \begin{tabular}{l|l} 
DOI: 10.1159/000430401 & (c) 2015 S. Karger AG, Basel
\end{tabular} www.karger.com/cpb \\ Gong et al.: Humanized Mice Model for Bladder Cancer}

26 Siegel RL, Miller KD, Jemal A: Cancer statistics, 2015. CA Cancer J Clin 2015;65:5-29.

27 Tan MY, Mu XY, Liu B, Wang Y, Bao ED, Qiu JX, Fan Y: SUMO-specific protease 2 suppresses cell migration and invasion through inhibiting the expression of MMP13 in bladder cancer cells. Cell Physiol Biochem 2013;32:542-548.

28 Gabriel U, Bolenz C, Michel MS: Experimental models for therapeutic studies of transitional cell carcinoma. Anticancer Res 2007;27:3163-3171.

29 Chan ES, Patel AR, Smith AK, Klein JB, Thomas AA, Heston WD, Larchian WA: Optimizing orthotopic bladder tumor implantation in a syngeneic mouse model. J Urol 2009;182:2926-2931.

30 Talmadge JE, Singh RK, Fidler IJ, Raz A: Murine models to evaluate novel and conventional therapeutic strategies for cancer. Am J Pathol 2007;170:793-804.

31 Sartelet H, Durrieu L, Fontaine F, Nyalendo C, Haddad E: Description of a new xenograft model of metastatic neuroblastoma using NOD/SCID/Il2rg null (NSG) mice. In Vivo 2012;26:19-29.

32 Pearson T, Greiner DL, Shultz LD: Humanized SCID mouse models for biomedical research. Curr Top Microbiol Immunol 2008;324:25-51.

33 Shpitz B, Chambers CA, Singhal AB, Hozumi N, Fernandes BJ, Roifman CM, Weiner LM, Roder JC, Gallinger S: High level functional engraftment of severe combined immunodeficient mice with human peripheral blood lymphocytes following pretreatment with radiation and anti-asialo GM1. J Immunol Methods 1994;169:115.

34 Hochman PS, Cudkowicz G, Dausset J: Decline of natural killer cell activity in sublethally irradiated mice. J Natl Cancer Inst 1978;61:265-268.

35 Barry TS, Jones DM, Richter CB, Haynes BF: Successful engraftment of human postnatal thymus in severe combined immune deficient (SCID) mice: differential engraftment of thymic components with irradiation versus anti-asialo GM-1 immunosuppressive regimens. J Exp Med 1991;173:167-180.

36 Han C, Zheng B, Tian J, Ji J, Xu Z, Hou H: Superantigen-induced cytotoxic T lymphocytes suppress growth of bladder carcinoma cells in vitro and in vivo. Zhonghua Zhong Liu Za Zhi 2000;22:392. Chinese.

37 Shibina LV, Svitich OA, Krasnoproshina LI, Skhodova SA, Ordiiants IM, Bisheva IV: Expression of TLR2 and TLR9 genes by epithelial cells of cervical canal mucous membrane in women with inflammatory diseases of small pelvis organs. Zh Mikrobiol Epidemiol Immunobiol. 2013;3:68-72.

38 Nadella MV, Kisseberth WC, Nadella KS, Thudi NK, Thamm DH, McNiel EA, Yilmaz A, Boris-Lawrie K, Rosol TJ: NOD/SCID mouse model of canine T-cell lymphoma with humoral hypercalcaemia of malignancy: cytokine gene expression profiling and in vivo bioluminescent imaging. Vet Comp Oncol 2008;6:39-54.

39 Erdmann N, Bondarenko A, Hewicker-Trautwein M, Angrisani N, Reifenrath J, Lucas A, Meyer-Lindenberg A: Evaluation of the soft tissue biocompatibility of $\mathrm{MgCa} 0.8$ and surgical steel $316 \mathrm{~L}$ in vivo: a comparative study in rabbits. Biomed Eng Online 2010;9:63.

40 Bondarenko A, Hewicker-Trautwein M, Erdmann N, Angrisani N, Reifenrath J, Meyer-Lindenberg A: Comparison of morphological changes in efferent lymph nodes after implantation of resorbable and nonresorbable implants in rabbits. Biomed Eng Online 2011;10:32.

41 Tsumori K, Kohrogi H, Goto E, Hirata N, Hirosako S, Fujii K, Ando M, Kawano O, Mizuta H: T cells of atopic asthmatics preferentially infiltrate into human bronchial xenografts in SCID mice. J Immunol 2003;170:5712-5718.

42 Iwanuma Y, Chen FA, Egilmez NK, Takita H, Bankert RB: Antitumor immune response of human peripheral blood lymphocytes coengrafted with tumor into severe combined immunodeficient mice. Cancer Res 1997;57:2937-2942.

43 Sabzevari H, Reisfeld RA: Human cytotoxic T-cells suppress the growth of spontaneous melanoma metastases in SCID/hu mice. Cancer Res 1993;53:4933-4937.

44 Pulliero A, Marengo B, Domenicotti C, Longobardi MG, Fazzi E, Orcesi S, Bianchi M, Balottin U, Izzotti A: Inhibition of neuroblastoma cell growth by TREX1-mutated human lymphocytes. Oncol Rep 2012;27:16891694.

45 Brehm MA, Shultz LD, Greiner DL: Humanized mouse models to study human diseases. Curr Opin Endocrinol Diabetes Obes 2010;17:120-125. 